\title{
Analysis Acceleration in the Design of Frequency Selective Surfaces using Parametric Techniques
}

\author{
Dave J. Bekers ${ }^{\# 1}$, Stefania Monni ${ }^{\# 2}$, Vincent Ducros ${ }^{* 3}$, Giampiero Gerini ${ }^{\# 4}$, Michel Rochette ${ }^{* 5}$ \\ \# TNO Defense, Security and Safety, \\ Oude Waalsdorperweg 63, PO Box 96864, 2509 JG The Hague, The Netherlands

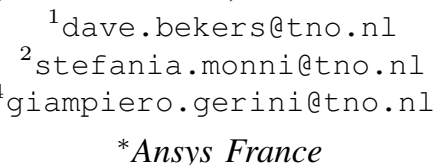 \\ Immeuble Einstein 11, Boulevard Einstein, F-69100 Villeurbanne, France \\ 3 vincent.ducrosdansys.com \\ ${ }^{5}$ michel.rochettedansys.com
}

\begin{abstract}
The design of multi-layer Frequency Selective Surfaces often requires characterising the behaviour of the structure in wide frequency and angle ranges. Moreover, a fine tuning of the design leads to a large parameter space and results in a long computational time. To address this problem, an efficient parameterisation and interpolation technique has been implemented within the Multimode Equivalent Network approach based on the Integral Equation formulation, a technique developed for the analysis of multi-layer periodic structures. The paper describes this technique and shows for a test case consisting of an array integrated with an FSS how the calculation time can be drastically reduced.
\end{abstract}

\section{INTRODUCTION}

A Frequency Selective Surface (FSS) is an array of periodically arranged metallic patches or apertures cut on a metallic plate, designed to obtain a certain filtering behaviour with respect to the frequency and/or the angle of incidence of the impinging electromagnetic wave. FSS performances are characterised in terms of reflection and transmission coefficient, calculated by forcing the appropriate boundary condition at each element and solving the corresponding integral equation. For a multi-layer structure, consisting of several FSSs sandwiched between dielectric layers, the scattering problem can be addressed either by solving the corresponding system of coupled integral equations or by resorting to an equivalent microwave network representation of each layer and then cascading all the networks to derive a global representation of the whole structure. Because of its modularity, the latter approach is particularly convenient as basis for the design. In particular, the Multimode Equivalent Network approach based on the Integral Equation formulation (IEMEN) [1] allows analysing multi-layer FSSs integrated with waveguide phased arrays taking also into account feeding and filtering elements inside the array. Usually, the equivalent networks have as many input and output ports as the number of modes used to represent the fields at the transition. To reduce computational time and to avoid stability problems [2], the IEMEN method resorts to an equivalent network (represented by an impedance/admittance matrix) in terms of only accessible modes. They are the modes (propagating or evanescent) used in the representation of the field at the FSS transition that also contribute to the interaction with adjacent FSS transitions. The scattering problem is formulated in terms of an integral equation with reduced kernel and multiple forcing terms, one for each accessible modes [3]. The equation is solved by the Method of Moments with a Galerkin formulation.

The IEMEN approach has been successfully applied to the design of several FSSs. Requirements set on the FSS performances usually need the evaluation of reflection and transmission coefficients for different scan-angle and scanplane configurations in the given operating frequency range. A first design phase, based for example on the use of equivalent circuits [1], usually results in the identification of the appropriate FSS element type and multi-layer configuration. A fine tuning on the element size and on the thickness of the dielectric layers is required in order to optimise the performances of the structure with respect to the requirements. Hence, a large number of simulations needs to be carried out, which makes this phase very time consuming.

In general, an efficient design should be based on a interactive procedure. For this purpose, a parametrisation and interpolation technique has been implemented which greatly reduces the calculation time. In Sec. II the technique is outlined and in Section III it is applied to a first test case consisting of a waveguide phased array cascaded to a dipole FSS. More complex test cases, including a multi-layer FSS, will be described in the oral presentation. Conclusions are drawn in Sec. IV.

\section{PARAmetric AND Interpolation TEChNiQues}

The most time-consuming step in the IEMEN approach is the discretisation of the integral equations that describe the radiating structures, i.e., patches and slots, in the FSS. The computation of the corresponding moment matrices require the evaluation of a spectral sum for each matrix component. The next steps in the approach, i.e., solving the moment matrix systems of the radiating structures, the construction of 
the mode matrices (impedance/admittance) of all layers and radiating structures, and the cascading of these matrices, take much less computation time. Hence, in order to accelerate the analysis of FSSs by the IEMEN approach, we need in particular to accelerate the computation of the moment matrices.

Given a large parameter space of frequency $f$, scan angles $\phi$ and $\theta$, and shape parameters $p_{1}, \ldots, p_{N}$ for a radiating structure, we can reduce the number of moment-matrix evaluations by the application of parametric and interpolation techniques. Such techniques can also be applied on the level of the mode matrices of the radiating structures or even on the level of the mode matrix of the complete FSS. This seems very appealing from a computational point of view, since it reduces not only the number of moment-matrix evaluations, but also the number of mode matrix evaluations and the number of cascade operations. On the other hand, application of such techniques to the moment matrices of the radiating structures allows for a greater geometrical flexibility. By removing basis functions from the moment matrices, we can straightforwardly alter the geometry of the radiating structures. Such an operation is impossible with interpolated mode matrices. Given the low computational time of the mode matrices and the cascade operations, we choose to apply parametric and interpolation techniques to the moment matrices of the radiating structures.

We explain the parametric techniques in more detail. Let the moment-matrix equation be given by

$$
\begin{aligned}
Z\left(f, \theta, \phi, p_{1}, \ldots, p_{N}\right) I\left(f, \theta, \phi, p_{1}, \ldots, p_{N}\right) & = \\
& =V\left(f, \theta, \phi, p_{1}, \ldots, p_{N}\right),
\end{aligned}
$$

where all parameters have prescribed ranges. We cast the matrix and the right-hand side (RHS) of this equation in a row vector, $[Z, V]$, by putting first the (transposed) columns of $Z$ one after the other and, next, the (transposed) RHS. To any parameter point $\left(f, \theta, \phi, p_{1}, \ldots, p_{N}\right)$ in the specified ranges corresponds a row vector $\left[Z\left(f, \theta, \phi, p_{1}, \ldots, p_{N}\right), V\left(f, \theta, \phi, p_{1}, \ldots, p_{N}\right)\right]$. Let $\mathcal{S}$ be the space of all row vectors. Then, the problem of generating rapidly the moment matrix $Z$ and the RHS $V$ for any parameter point $\left(f, \theta, \phi, p_{1}, \ldots, p_{N}\right)$ in the specified ranges is equivalent to generating rapidly any vector in $\mathcal{S}$. To accomplish this task, we employ the following strategy.

We first construct a basis for $\mathcal{S}$ in a gradual way. To this end, we select an initial set of paramter points, typically 5 points, for which we generate the corresponding vectors $[Z, V]$ by full-wave computations, as described above. On the obtained set of vectors, we perform a singular-value analysis. Next, we extend the set of parameter points, typically by 5 points, and generate the vectors $[Z, V]$ for the added points. Subsequently, we perform a singular-value analysis on the extended set of vectors $[Z, V]$. By comparing both singular-value analyses, we determine whether the amount of vectors is sufficient to span $\mathcal{S}$. We repeat the process of extending the set of parameter points until the amount of vectors is sufficient. These vectors represent a basis for $\mathcal{S}:\left[Z_{m}, V_{m}\right], m=1, \ldots, M$.
After construction of the basis, we determine the subspaces of the space of parameter points in which the Green's kernel of the radiating structure is differentiable. Only if the kernel is differentiable, interpolation of the casted moment matrix and RHS $[Z, V]$ will make sense. The subspaces are called differentiability zones. At each zone boundary a specific mode turns into propagation. In each differentiability zone, we compute expansion coefficients $\alpha_{m}$ that describe the row vector $[Z, V]$ in terms of the basis elements $\left[Z_{m}, V_{m}\right]$ inside the zone,

$$
\begin{aligned}
& {\left[Z\left(f, \theta, \phi, p_{1}, \ldots, p_{N}\right), V\left(f, \theta, \phi, p_{1}, \ldots, p_{N}\right)\right] \approx} \\
& \approx \sum_{m=1}^{M} \alpha_{m}\left(f, \theta, \phi, p_{1}, \ldots, p_{N}\right)\left[Z_{m}, V_{m}\right]
\end{aligned}
$$

The computation of these coefficients is carried out by means of Kriging interpolation over a gradually extended set of parameter points $\left(f, \theta, \phi, p_{1}, \ldots, p_{N}\right)$ in the zone. For each considered set, the Kriging results are compared with results obtained by reduced order model techniques applied to the minimum norm problem associated to (2). The extension of the set stops once a required level of accuracy of the expansion coefficients $\alpha_{m}$ is achieved.

The described strategy is called the parametric computation. After this computation, the moment matrix and the RHS for any parameter point in the specified parameter ranges can be rapidly evaluated by simply interpolating the expansion coefficients $\alpha_{m}$ and by calculating the sum (2). This evaluation, which we call post processing, is much faster than the fullwave computation. Hence, it offers a rapid 'walk' through the parameter space and, therewith, it opens the door for the optimization of FSS designs in a much more efficient way than by full-wave computations.

With respect to the parametric computation itself, we note that only in the construction of the basis, full-wave computations of the complete moment matrices are required. In general, the number of matrices $M$ in the basis is much lower than the number of parameter points in which one would like to evaluate the scattering parameters of the FSS. Moreover, the reduced order model techniques and the Kriging interpolation are much less computationally expensive than the computation of all moment matrices.

\section{TEST CASES}

The parametrisation technique described in Sec. II has been applied to a dipole FSS cascaded to a waveguide phased array. The unit-cell geometry is illustrated in Fig. 1.

The waveguides have transverse dimensions $12 \mathrm{~mm} \times 6 \mathrm{~mm}$ and are filled with dielectric material of permittivity $\varepsilon_{r}=$ 3.38. The FSS dipoles have length $\mathrm{L}=6 \mathrm{~mm}$ and width $\mathrm{W}=$ $1 \mathrm{~mm}$. They are arranged on a rectangular lattice of dimensions $15 \mathrm{~mm} \times 10 \mathrm{~mm}$, on a slab of foam of dielectric constant $\varepsilon_{r}=1.03$ and thickness $15 \mathrm{~mm}$. The waveguide is excited by the TE10 mode. Because of the large distance between FSS and array, one accessible mode (the fundamental Floquet mode) was sufficient to perform the analysis using the IEMEN 


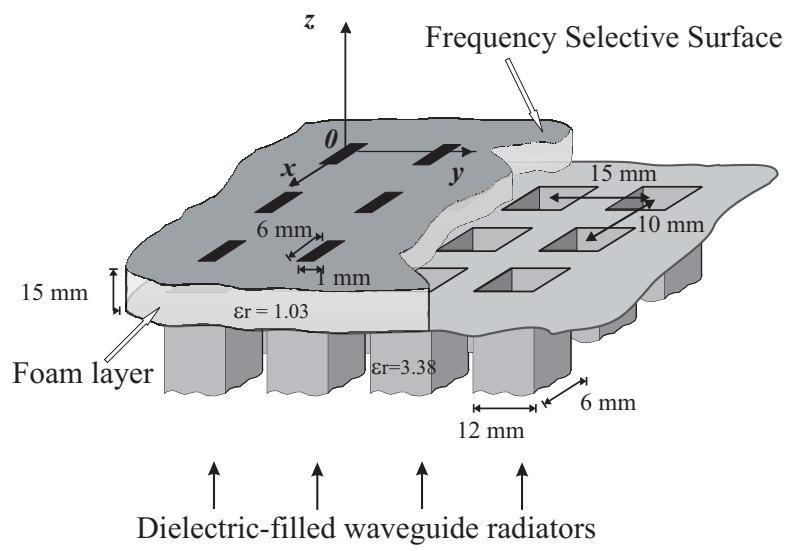

Fig. 1. Unit cell geometry of the test case: dipole FSS cascaded to a waveguide phased array.

TABLE I

PARAMETER VALUES FOR PARAMETRIC COMPUTATION.

\begin{tabular}{|l|l|l|}
\hline Parameters & Lower bound & Upper bound \\
\hline Frequency $f$ & $8 \mathrm{GHz}$ & $12 \mathrm{GHz}$ \\
\hline Scan angle $\theta$ & $0^{\circ}$ & $30^{\circ}$ \\
\hline Scan angle $\phi$ & $10^{\circ}$ & $10^{\circ}$ \\
\hline Dipole size $\mathrm{L} \times \mathrm{W}$ & $6 \mathrm{~mm} \times 1 \mathrm{~mm}$ & $9 \mathrm{~mm} \times 1.5 \mathrm{~mm}$ \\
\hline
\end{tabular}

approach. The equivalent electric currents on the dipole were discretised in terms of 24 piece wise linear functions, resulting in a $24 \times 24$ moment matrix and a $24 \times 1$ RHS. Consequently, $[Z, V]$ is a row vector with $24 \times 24+24 \times 1=600$ elements.

The parameters considered for this example are frequency, scan angles and dipole size. Table I shows the parameter values for the parametric computation. Note that length and width of the dipole are changed simultaneously. The CPU time needed for the parametric computation is $51 \mathrm{~min}$. The CPU time of the post processing is only $0.15 \mathrm{sec}$ per parameter point, while the CPU time of a full-wave computation is $148 \mathrm{sec}$ per parameter point. Although the parametric computation seems expensive, only 21 full-wave computations can be carried out in $51 \mathrm{~min}$. On the contrary, having performed the parametric computation, the post processing in any parameter point in the ranges of Table I requires only $0.15 \mathrm{sec}$, which is a factor 1000 less than the cost of a full-wave computation.

Fig. 2 shows the relative difference in S11 between the postprocessing and the full-wave results, both for the smallest dipole size and for the largest dipole size. For the smallest dipole size, the differences are smaller than $-30 \mathrm{~dB}$ for all frequencies and elevation angles. For the largest dipole size, the differences are smaller than $-20 \mathrm{~dB}$, except at $12 \mathrm{GHz}$, where the difference is around $-10 \mathrm{~dB}$. These higher differences are explained by the presence of a resonance at $11.6 \mathrm{GHz}$. To investigate the accuracy of the interpolation in more detail, we ran an additional post processing for the scan angles $\theta=10^{\circ}$ and $\theta=20^{\circ}$, and for 41 frequency points instead of 11. Fig. 3 shows the S11 obtained by full-wave computation and by parametrisation and post processing. Even near the resonance,

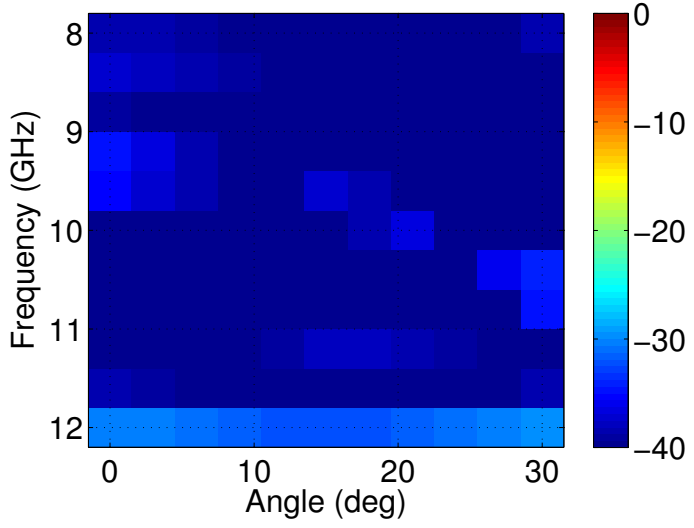

(a)

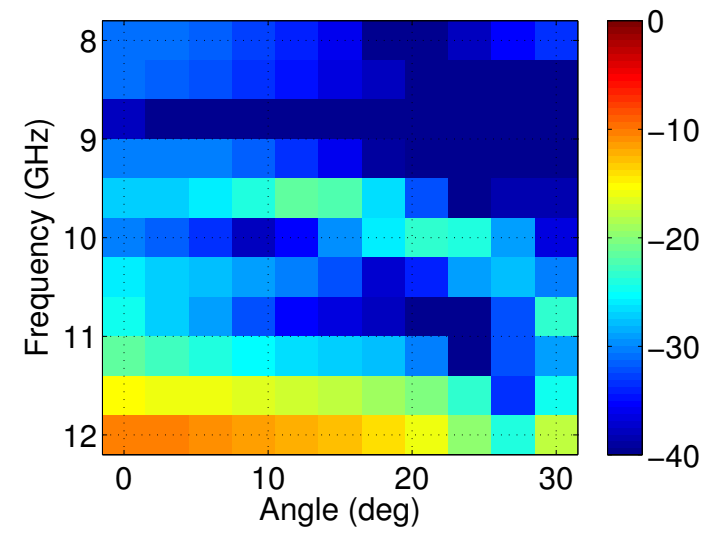

(b)

Fig. 2. Relative difference in S11 between the full-wave and the postprocessing results obtained using the data set in Table I. (a): First shape parameter point $(\mathrm{L}=6 \mathrm{~mm}, \mathrm{~W}=1 \mathrm{~mm})$. (b): Second shape parameter point $(\mathrm{L}=9 \mathrm{~mm}, \mathrm{~W}=1.5 \mathrm{~mm})$.

the accuracy of the interpolation is remarkable.

\section{Conclusions}

The fine tuning of a multi-layer FSS design requires assessing the FSS performances for variations of some key parameters (e.g. FSS dimensions and/or separation between consecutive layers) in the operating frequency range and for all given angle configurations. Consequently, a large amount of simulations has to be carried out, which results in high computational times. In this paper an efficient parameterisation and interpolation technique for the acceleration of FSS simulations is outlined. The technique has been applied to a software tool based on the IEMEN approach and allows for a drastic reduction of the total computational time. The application of the technique is illustrated by a test case consisting of an FSS cascaded with a waveguide phased array. Other test cases, 
including a multi-layer FSS, will be shown during the oral presentation.

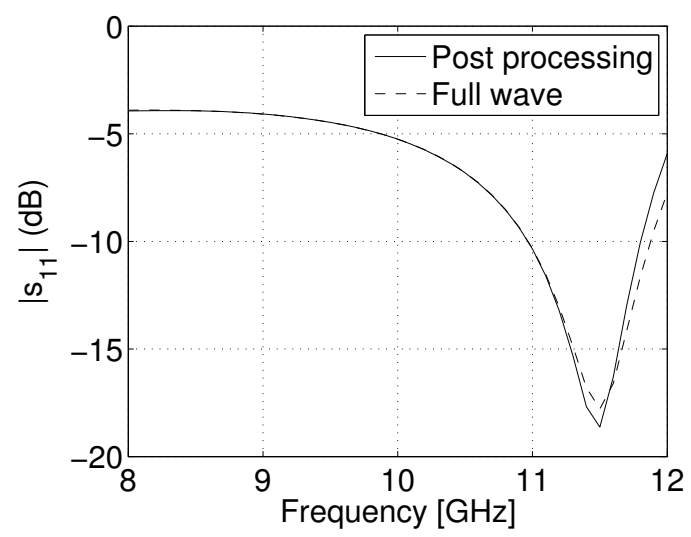

(a)

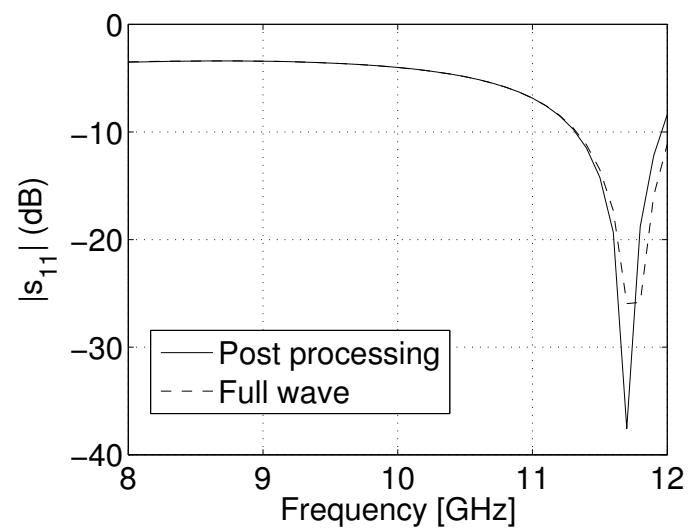

(b)

Fig. 3. S11 of the structure of Fig 1: comparison between the full-wave results and the post-processing results obtained using the data set in Table I, considering the shape parameter point $\mathrm{L}=9 \mathrm{~mm}, \mathrm{~W}=1.5 \mathrm{~mm}$, for (a) scan angle $\theta=10^{\circ}$ and (b) scan angle $\theta=20^{\circ}$.

\section{ACKNOWLEDGMENT}

The work described in this paper was carried out in the frame of the European project SYNTAS in which Thales Hengelo (The Netherlands), Thales Airborne Systems (France), Ansys France, and TNO Defense, Security and Safety participate.

\section{REFERENCES}

[1] S. Monni, "Frequency Selective Surfaces Iintegrated with Phased Array Antennas: Analysis and Design using Multimode Equivalent Networks," Ph.D. dissertation, Technical University of Eindhoven, Eindhoven, The Netherlands, 2005.

[2] F. Alessandri, G. Bartolucci, and R. Sorrentino, "Admittance matrix formulation of waveguide discontinuity problems: computer-aided design of branch guide directional couplers," IEEE Trans. Microwave Theory and Techniques, vol. 36, no. 2, pp. 394-403, 1988.
[3] S. Monni, G. Gerini, A. Neto, and A. G. Tijhuis, "Multimode equivalent networks for the design and analysis of frequency selective surfaces," IEEE Trans. Antennas and Propagation (submitted for publiaction). 